\title{
Echocardiography in infective endocarditis
}

\author{
A Evangelista, M T González-Alujas
}

Heart 2004;90:614-617. doi: 10.1136/hrt.2003.029868

S ince Dillon and colleagues ${ }^{1}$ in 1973 described valvar vegetations by $M$ mode echocardiography, the technique has assumed an increasingly important role in the assessment and management of endocarditis. The development of two dimensional and later transoesophageal echocardiography (TOE) has significantly improved the non-invasive detection of vegetations. Moreover, echoDoppler studies provide clinically important information on the presence and degree of valvar destruction and their haemodynamic consequences, as well as on the existence of perivalvar infection. The diagnostic strategy proposed by Durack and colleagues ${ }^{2}$ (the Duke criteria) combined echocardiographic findings with clinical and microbiological data. Three echocardiographic findings were considered to be major criteria for the diagnosis of endocarditis: (1) presence of vegetations defined as mobile echodense masses implanted in a valve or mural endocardium in the trajectory of a regurgitant jet or implanted in prosthetic material with no alternative anatomical explanation; (2) presence of abscesses; or (3) presence of a new dehiscence of a valvar prosthesis. Abnormal echocardiographic findings not fulfilling those definitions were considered minor criteria. More recently the use of TOE has resulted in better imaging and therefore doubtful findings are not considered minor criteria any longer. ${ }^{3}$ Since the definite diagnosis of endocarditis requires the presence of two major criteria, or one major and three minor criteria, it is clear that echocardiography has assumed a crucial role in the diagnosis of the disease, particularly when blood cultures are negative.

\section{DETECTION OF VEGETATIONS}

There is no better technique for non-invasive visualisation of vegetations than echocardiography (fig 1). Overall the detection rate for vegetations by transthoracic echocardiography (TTE) in patients with a clinical suspicion of endocarditis averages around $50 \%{ }^{4}$ The diagnostic yield of the technique in the detection of vegetations is influenced by several factors: image quality; echogenicity and vegetation size; vegetation location; presence of previous valvar disease or valvar prosthesis; experience and skill of the examiner; and pre-test probability of endocarditis.

\section{Native valve endocarditis}

The use of harmonic imaging has improved study quality, but not the sensitivity in the visualisation of vegetations. ${ }^{45}$ Vegetation size also affects TTE sensitivity ${ }^{6}$ since only $25 \%$ of vegetations $<5 \mathrm{~mm}$ and $70 \%$ of those between $6-10 \mathrm{~mm}$ are identified. ${ }^{7}$ Underlying valve disease may influence the diagnostic accuracy of TTE when a myxomatous mitral valve or sclerotic or calcified valves are present. These limitations have been overcome by TOE owing to its better resolution and multiple study planes. Many studies have compared the sensitivity and specificity of TTE and TOE in the diagnosis of vegetations (table 1 ). In the majority of these studies TTE sensitivity ranges between $40-63 \%$ and that of TOE between $90-100 \%{ }^{4}{ }^{6-8}$
A negative TOE has an important clinical impact on the diagnosis of endocarditis with a high negative predictive value ranging from $86-97 \%$. In patients with native heart valves a negative TOE virtually rules out the diagnosis of infective endocarditis. However, in the study of Sochowski and colleagues ${ }^{9}$ five out of 65 patients $(7.6 \%)$ with an initially negative TOE were finally diagnosed with endocarditis: in three of the patients a TOE performed 1-2 weeks after the initial examination showed the presence of vegetations. This study underlines the importance of recognising the phase of the disease in which the study is performed, since vegetations may not be large enough to be visualised when endocarditis is suspected very early on.

In common with other diagnostic techniques, specificity of echocardiography is related to the clinical indication and the type of population studied. The technique does not permit differentiation between septic vegetations and other aseptic vegetations present in other situations such as Libman-Sacks endocarditis in systemic lupus erythematosus, and antiphospholipid syndrome or marantic endocarditis. Vegetations persisting after effective treatment must not be interpreted as a clinical recurrence of the disease unless supported by clinical features and bacteriologic evidence. Specificity is influenced mainly by the presence of valvar thickening or calcifications and also by rupture mitral chordae tendinae or Lambl's nodules which can be confused with vegetation images. ${ }^{4} 8$

The usefulness of TOE in patients with suspected endocarditis on a native valve is related to the results of the TTE. TOE is useful when TTE is negative or inconclusive.

\section{Prosthetic valve endocarditis}

Vegetations on prosthetic valves are more difficult to detect by TTE than those involving native valves, and therefore TOE should always be used if the diagnosis of prosthetic endocarditis is suspected (fig 2). The sewing ring and support structures of mechanical and bioprosthetic valves are strongly echogenic and may prevent vegetation detection within the valve apparatus or its shadow. The vegetative growth appears as thickening and irregularity of the normally smooth contour of the sewing ring. Both thrombus and pannus have a similar appearance and cannot be distinguished from vegetative material. It is also important to recognise strands to avoid false positive diagnoses. Strands are commonly observed by TOE on prosthetic valves, particularly in the early postoperative months, especially in mitral prostheses. ${ }^{10}$

In large series of prosthetic endocarditis TOE has shown an 86-94\% sensitivity and $88-100 \%$ specificity for vegetation diagnosis, while TTE sensitivity was only $36-69 \%{ }^{11}$ Bioprosthetic valve leaflets may become infected with secondary destruction of leaflet tissue. The distinction between wear-and-tear degeneration of tissue valves and endocarditis is often difficult. TOE also led to an improved diagnostic accuracy in the diagnosis of endocarditis on bioprosthetic valves ${ }^{8}$ 


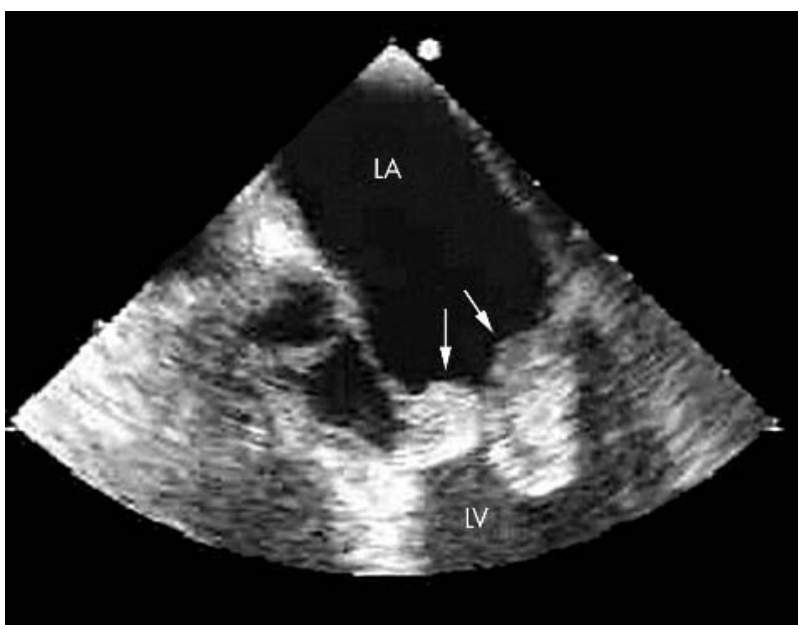

Figure 1 Large vegetations (arrows) in the mitral valve secondary to fungal endocarditis. LA: left atrium; LV: left ventricle.

\section{Right sided endocarditis and pacemaker lead infections}

TTE allows an easy and correct diagnosis of tricuspid vegetations, probably because the majority of patients with tricuspid endocarditis are young intravenous drug abusers with large vegetations. The vegetations are located in the atrial side of the tricuspid valve, in the way of the regurgitant jet. San Roman and colleagues ${ }^{12}$ showed that TOE did not increase the accuracy of TTE in the detection of vegetations in tricuspid endocarditis. However, despite the low number of cases described, TOE appears to be more sensitive than TTE in the diagnosis of pulmonary valve endocarditis.

Infection or endocarditis on a pacemaker lead are difficult to diagnose by TTE since pacemaker leads produce reverberations and artefacts that may mask or make difficult the recognition of vegetations close to these structures. In addition when vegetations were visualised it was difficult to determine whether tricuspid valve endocarditis, lead infection, or both were present. TOE was clearly superior to TTE in this clinical setting (sensitivity 23\% v 94\%).

\section{DIAGNOSIS OF COMPLICATIONS}

The infective organism within the vegetation may cause destruction of the underlying valvar tissue and this process can extend to produce perivalvar complications.

\section{Valvar complications}

Regurgitation of the infected valve is almost constant and results from a variety of mechanisms. The vegetations themselves may prevent proper leaflet or cusp coaptation. Some degree of valvar destruction is commonly seen and may vary from a small perforation in a cusp to a complete flail leaflet. Valvar perforation is a frequent complication that may cause severe insufficiency with an acute onset and precipitate

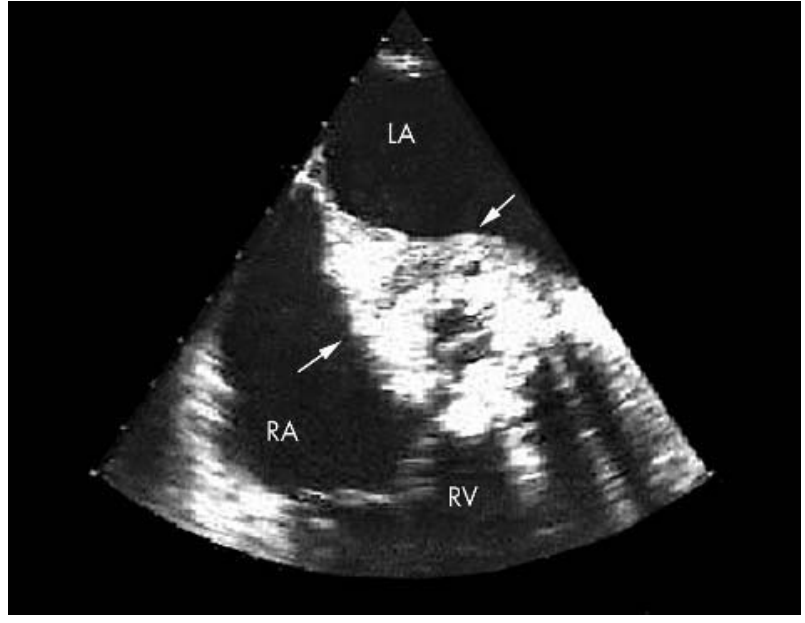

Figure 2 Aortic periannular abscess (arrows) in a prosthetic valve endocarditis. LA: left atrium; RA: right atrium; RV: right ventricle.

heart failure. In aortic endocarditis cusp perforation, flail or both may occur in up to $50 \%$ of cases. Severe aortic insufficiency as estimated by Doppler has been associated with poor prognosis. In this setting early diastolic closure of the mitral valve identifies patients with an unstable haemodynamic status. Perforation of the mitral leaflets is less common, occurring in only $15 \%$ of the patients with mitral valve endocarditis. Progressive destruction of the mitral valve results initially in ruptured chordae tendinae and ultimately flail leaflet.

TTE appears more useful in detecting mitral than aortic perforations. Colour flow Doppler imaging allows location of abnormal flows at the areas of anatomic interruption and therefore it helps to differentiate mitral cusp perforation from true mitral regurgitation. TOE is recommended if a valve perforation is suspected and TOE is negative or equivocal.

TOE colour flow mapping is of particular value in patients with a mechanical mitral prosthesis and paravalvar regurgitation. The presence of a new or increasing paravalvar regurgitation or valve dehiscence is a major criteria for the diagnosis of endocarditis. Khanderia and colleagues ${ }^{13}$ reported that the transoesophageal approach had an overall sensitivity of $96 \%$ in the evaluation of mitral prostheses. The demonstration of aortic prosthetic paravalvar regurgitation is rather easy from the precordium as the colour encoded regurgitant jets may be visualised from both the apical and parasternal views.

\section{Perivalvar complications}

Extension of the infection to the perivalvar tissues is a sign of poor prognosis in the evolution of the disease. Extravalvar extension may lead to endothelial erosion, perivalvar abscess, mycotic aneurysm, and intracardiac fistulae.

Table 1 Studies comparing the sensitivity and specificity of transthoracic echocardiography (TTE) and transoesophageal echocardiography (TOE) in the diagnosis of vegetations

\begin{tabular}{|c|c|c|c|c|c|}
\hline & \multirow[b]{2}{*}{ No. } & \multicolumn{2}{|c|}{ Sensitivity (\%) } & \multicolumn{2}{|c|}{ Specificity (\%) } \\
\hline & & TIE & TOE & TTE & TOE \\
\hline $\begin{array}{l}\text { Shapiro et al } l^{6} 1994 \\
\text { Erbel et al } 1988 \\
\text { Shively et al } \\
8^{8} 1991\end{array}$ & $\begin{array}{l}64 \\
96 \\
66\end{array}$ & $\begin{array}{l}60 \\
63 \\
44\end{array}$ & $\begin{array}{r}87 \\
100 \\
94\end{array}$ & $\begin{array}{l}91 \\
98 \\
98\end{array}$ & $\begin{array}{r}91 \\
98 \\
100\end{array}$ \\
\hline
\end{tabular}




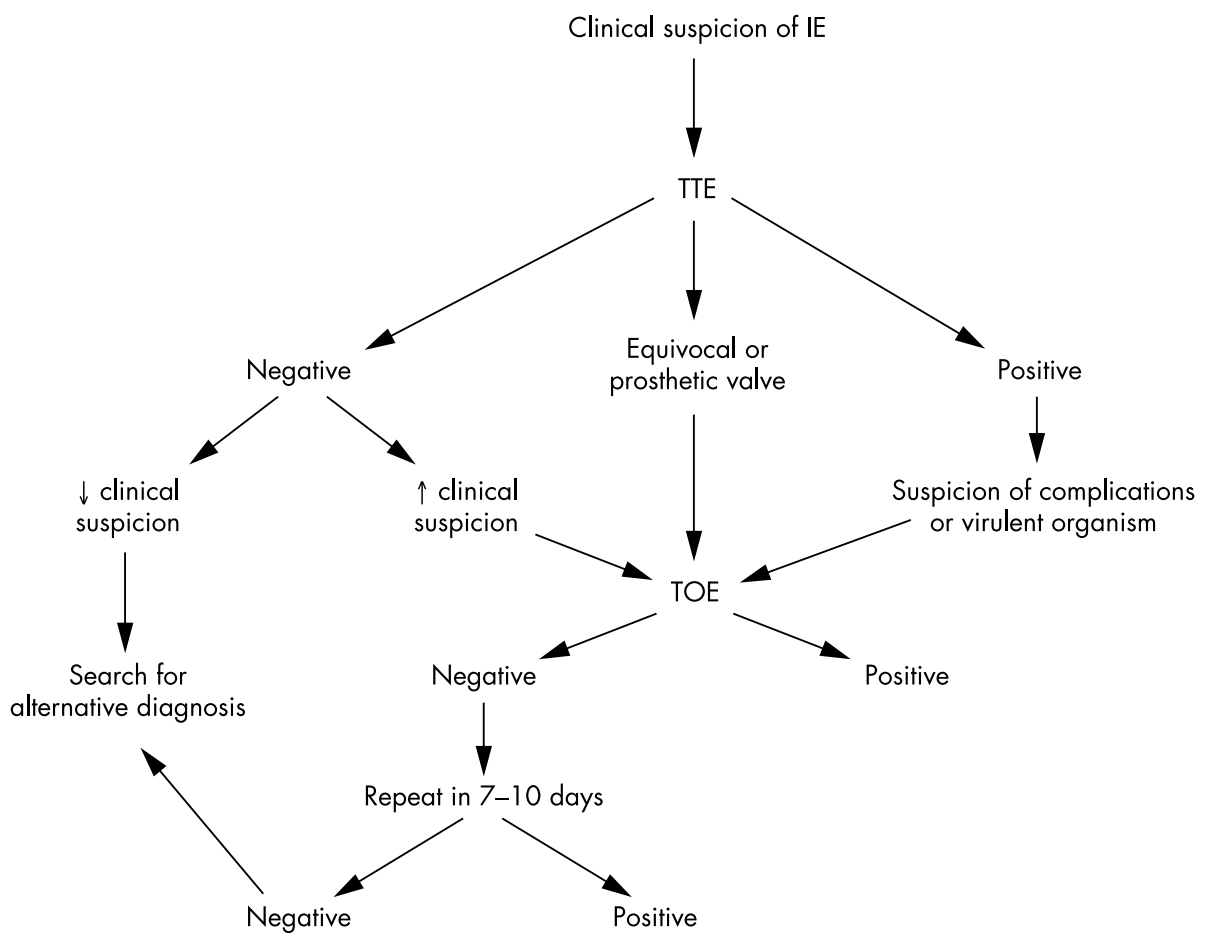

Figure 3 The diagnostic assessment in infectious endocarditis.

\section{Perivalvar abscess}

Perivalvar cavities are formed when annular infections break through and spread into contiguous tissue. In native aortic valve endocarditis they generally occur through the weakest portion of the annulus, which is near the membranous septum. Perivalvar abscesses are particularly common in prosthetic valve endocarditis, since the annulus is the usual primary site of infection. A perivalvar abscess is considered to be present when a definite region of reduced echo density is found on the echocardiogram, or when echolucent cavities within the valvar annulus or adjacent myocardial structures are found in the setting of valvar infection. Daniel and colleagues ${ }^{14}$ reported 44 patients with proven abscesses. Sensitivity and specificity of TTE for abscess detection were $28 \%$ and $99 \%$, respectively, compared with $87 \%$ and $95 \%$ with TOE. TOE was especially useful in prosthetic endocarditis. The diagnosis of aortic abscesses was easier than mitral abscesses, both with TTE (42\% v9\%) and TOE $(86 \% v 57 \%)$.

\section{Intervalvar fibrosa complications}

Secondary involvement of the mitral-aortic intervalvar fibrosa and anterior mitral leaflet occurs as a result of direct extension of the infection from the aortic valve or as a result of an infected aortic regurgitant jet. The abscess can expand to form a pseudoaneurysm and can subsequently cause a perforation and communication between the left ventricle and left atrium. An intervalvar pseudoaneurysm was defined as an echo-free cavity located posteriorly in the intervalvar fibrosa region, just below the aortic annulus, and bound by the base of the anterior mitral leaflet, the medial wall of the left atrium, and the posterior aortic root. Karalis and colleagues ${ }^{15}$ described 24 (44\%) complications involving 55 consecutive cases of aortic endocarditis, including eight abscesses and aneurysms in interfibrosa, seven interfibrosa perforations into the adjacent left atrium, and nine anterior mitral aneurysms and perforations. TTE detected $43 \%$ of these complications while TOE identified $90 \%$.

\section{Intracardiac fistulae}

Both aortic root abscesses and pseudoaneurysms may rupture into adjacent chambers and therefore create intracardiac fistulous tracts. These fistulae may be single or multiple and generally extend from the aorta to the right ventricle or the right or left atrium. ${ }^{16}$ Using colour Doppler the site of the communication of the ruptured intervalvar pseudoaneurysm is usually well defined. Using continuous wave Doppler, systolic high velocity flow suggests an abnormal communication between the aorta and either the left or right atria. Eccentric mitral regurgitation-type systolic jets by colour flow Doppler should suggest the possibility of interfibrosa perforation and the patient should therefore undergo further evaluation by TOE.

\section{CONCLUSIONS}

Any patient suspected of having infective endocarditis by clinical criteria should be screened by TTE. When the images are of good quality and the study is negative, an alternative diagnosis should be sought if the clinical suspicion is low. If the clinical suspicion is high TOE should be performed. TOE should also be performed if the results of the TTE are equivocal owing to underlying structural abnormalities or poor acoustic windows (fig 3).

If the TOE is negative, observation or re-evaluation of the clinical data are warranted. If the suspicion of endocarditis is high TOE should be repeated after 7-10 days to allow potential vegetations to become more apparent. A repeated negative study should virtually rule out the diagnosis unless TOE images are of poor quality.

TOE should also be performed to provide a more detailed anatomical assessment when there is a suspicion of perivalvar complications, particularly in the setting of aortic or prosthetic valve endocarditis, or in infections caused by virulent organisms such as Staphylococcus aureus.

\section{Authors' affiliations}

A Evangelista, M T Gonzalez-Alujas, Servicio de Cardiologia, Hospital Vall d' Hebron, Barcelona, Spain 
Correspondence to: $\operatorname{Dr}$ A Evangelista, Servicio de Cardiologia, Hospital Vall d' Hebron, $P^{\circ}$ Vall d' Hebron 119-129, 08035 Barcelona. Spain; evangel@hg.vhebron.es

\section{REFERENCES}

1 Dillon JC, Feigenbaum $\mathrm{H}$, Konecke LL, et al. Echocardiographic manifestations of valvular vegetations. Am Heart J 1973;6:698-704.

2 Durack DT, Lukes AS, Bright DK. New criteria for diagnosis of infective endocarditis: utilization of specific echocardiographic finding: Duke Endocarditis Service. Am J Med 1994;96:200-9.

3 Li JS, Suton DJ, Mick N, et al. Proposed modifications to the Duke criteria for the diagnosis of infective endocarditis. Clin Infect Dis 2000;30:633-8.

4 Mügge A, Daniel WG, Frank G, et al. Echocardiography in infective endocarditis: reassessment of prognostic implications of vegetation size determined by the transthoracic and the transesophageal approach. J Am Coll Cardiol 1989;14:631-8.

5 Reynolds HR, Jagen MA, Tunick PA, et al. Sensitivity of transthoracic versus transesophageal echocardiography for the detection of native valve vegetations in the modern era. J Am Soc Echocardiogr 2003;16:67-70.

6 Shapiro SM, Young E, De Guzman S, et al. Transesophageal echocardiography in diagnosis of infective endocarditis. Chest 1994; 105:377-82.

7 Erbel R, Rohmann S, Drexler M, et al. Improved diagnostic value of echocardiography in patients with infective endocarditis by transoesophageal approach. A prospective study. Eur Heart J 1988;9:43-53.
8 Shively BK, Gurule FT, Roldan CA, et al. Diagnostic value of transesophageal compared with transthoracic echocardiography in infective endocarditis. J Am Coll Cardiol 1991;18:391-7.

9 Sochowski RA, Chan K-L. Implication of negative results on a monoplane transesophageal echocardiographic study in patients with suspected infective endocarditis. J Am Coll Cardiol 1993;21:216-21.

10 Lengyel $M$. The impact of transesophageal echocardiography on management of prosthetic valve endocarditis: experience of 31 cases and review of the literature. J Heart Valve Dis 1997:6:204-11.

11 Rozich JD, Edwards WD, Hanna RD, et al. Mechanical prosthetic valve associated strands: Pathologic correlates to transesophageal echocardiography. J Am Soc Echocardiogr 2003;16:97-100.

12 San Roman JA, Vilacosta I, Zamorano JL, et al. Transesophageal echocardiography in right-sided endocarditis. J Am Coll Cardiol 1993;21:1226-30.

13 Khanderia BK, Seward JB, Oh JK, et al. Value and limitations of transesophageal echocardiography in assessment of mitral valve prostheses. Circulation 1991;83:1956-68.

14 Daniel WG, Mügge A, Martin RP, et al. Improvement in the diagnosis of abscesses associated with endocarditis by transesophageal echocardiography. N Engl J Med 1991;324:795-800.

15 Karalis DG, Bansal RC, Hauck AJ, et al. Transesophageal echocardiographic recognition of subaortic complications in aortic valve endocarditis. Circulation 1992;86:353-62.

16 Chan KL. Early clinical course and long-term outcome of patients with infective endocarditis complicated by perivalvular abscess. Can Med Assoc J 2002; 167:19-24.

\section{IMAGES IN CARDIOLOGY}

Improvement of cardiac hypertrophy and ventricular function in a man with Fabry disease by treatment with recombinant $\alpha$-galactosidase $A$

A 35 year old man was diagnosed as having Fabry disease because of extremely low activity of $\alpha$-galactosidase A and a point mutation of its gene. He had been suffering from acroparesthesias, angiokeratoma, corneal opacity, and hypohidrosis. Since his neuropathic pain was resistant to conventional treatment, he agreed with written informed consent to participate in an open label trial of intravenous recombinant $\alpha$-galactosidase A. He did not show overt heart or renal failure. However, he had cardiac hypertrophy and dysfunction. During six months of the trial, $0.2 \mathrm{mg} / \mathrm{kg}$ of $\alpha$-galactosidase A was administered intravenously every other week.

Echocardiography showed improvements in cardiac hypertrophy and systolic function: wall thickness decreased from $13 \mathrm{~mm}$ to $12 \mathrm{~mm}$, fractional shortening increased from $31 \%$ to $35 \%$, and left ventricular ejection fraction increased from $57 \%$ to $65 \%$ (panel 1). Furthermore, transmitral Doppler flow assessment confirmed improvements in diastolic function (panel 2). The peak flow velocity of the early filling (E) increased from $69 \mathrm{~cm} / \mathrm{s}$ to $97 \mathrm{~cm} / \mathrm{s}$, and the $\mathrm{E} / \mathrm{A}$ ratio increased from 1.1 to 1.6. Deceleration time of E decreased from $280 \mathrm{~ms}$ to $190 \mathrm{~ms}$. Magnetic resonance imaging also revealed improvements in cardiac hypertrophy and left ventricular function: wall thickness decreased from $15 \mathrm{~mm}$ to $12 \mathrm{~mm}$, left ventricular ejection fraction increased from $49 \%$ to $60 \%$, and left ventricular mass index decreased from $110.8 \mathrm{~g} / \mathrm{m}^{2}$ to $96.6 \mathrm{~g} / \mathrm{m}^{2}$ and normalised (panel 3). These findings show that repeated intravenous administration of $\alpha$-galactosidase A improved not only cardiac hypertrophy but also left ventricular systolic and diastolic function in Fabry disease.

\section{K Komamura \\ M Higashi \\ N Yamada \\ kkoma@hsp.ncvc.go.jp}
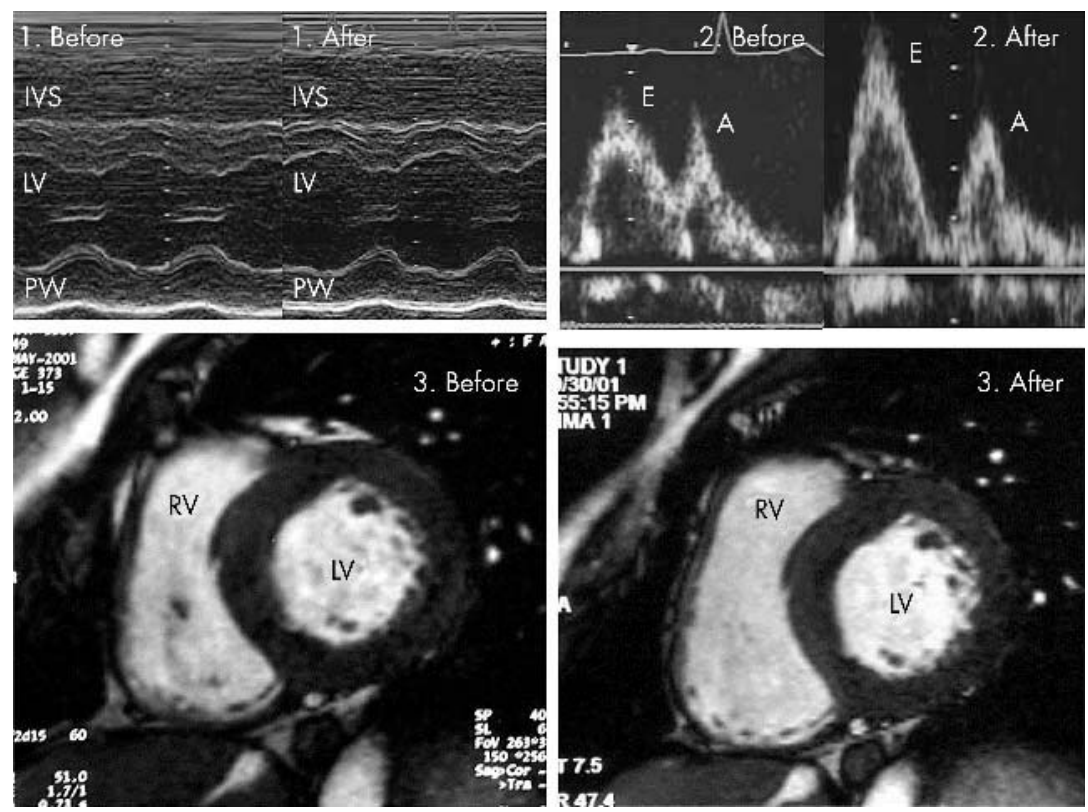